\title{
Padrões ecomorfológicos associados à dieta de Plagioscion squamosissimus (Perciformes, Scianidae) em reservatório permanente, no Nordeste do Brasil
}

\author{
Valdir P. Ferreira Filho¹, Thiago P. Guerraํ, Maria Cecília S. Lima², Daniela F. F. Teixeira ${ }^{4}$, Raquel R. \\ Costa $^{1}$, Isabela M. S. Araújo ${ }^{3}$, Ana Carla A. El-Deir ${ }^{1} \&$ Geraldo J. B. de Moura ${ }^{2}$
}

\footnotetext{
1. Programa de Pós-Graduação em Ecologia, Universidade Federal Rural de Pernambuco, Laboratório de Ecologia de Peixes, Rua Dom Manoel de Medeiros, s/n, 52171-900, Dois Irmãos, Pernambuco, Brasil.(valdir.paulo@gmail.com)

2. Programa de Pós-Graduação em Ecologia, Universidade Federal Rural de Pernambuco, Laboratório de Estudos Herpetológicos e Paleoherpetológicos, Rua Dom Manoel de Medeiros, s/n, 52171-900, Dois Irmãos, Pernambuco, Brasil.

3. Universidade Federal Rural de Pernambuco, Laboratório de Ictiologia, Departamento de Pesca e Aquicultura, Rua Dom Manoel de Medeiros, s/n, 52171-900, Dois Irmãos, Pernambuco, Brasil. 4. Universidade de Aveiro, Campus Universitário de Santiago 3810-193 Aveiro, Portugal.
}

\begin{abstract}
Ecomorphological patterns with diet of Plagioscion squamosissimus (Perciformes, Scianidae) in permanent reservoir in northeastern Brazil. The fish feeding and the ecomorphological patterns are related to several factors such as ontogeny. The present report was realized to test the hypothesis that the diet and the ecomorphological patterns present positive correlations between the different ontogeny stages of Plagioscion squamosissimus (Heckel, 1840) because as the species pass the young to adult phase to alter their diet and morphology. The croaker was chosen because is the more abundant specie in reservoir of Tapacurá Ecologic Station, state of Pernambuco. The ecomorphological patterns were valued and related with the feeding of individuals captured in a reservoir in April 2013. The morphometric measures were taken of all captured individuals and after were collected their stomachs. Fish were separated according to development stage, in juveniles and adults. Also total weight and their standard length were measured and eleven linear measurements were taken, used to obtain the indices that represent ecomorphological attributes. The food items were identified and separated until the smaller taxonomic level, been the most abundant the shrimp Macrobrachium amazonicum (Heller, 1862). It was observed that in the juveniles the items ingest presented wide amplitude size than adults. Through morphometric data it was observed that the head width values are bigger in juveniles and the body weight values are bigger in adults, being this important factors to determine the prey size ingested by the fish. It is concluded that the specie Plagioscion squamosissimus showed the relationship positive between morphology and feeding along the ontogenetic development evidenced by the variation in length of the ingest items between juveniles and adults, but there was no distinction in relation to ingest food between them.
\end{abstract}

KEYWORDS. Croakers, trophic ecology, ecomorphology, Tapacurá Ecological Station.

RESUMO. A alimentação de peixes e os padrões ecomorfológicos estão relacionados com vários fatores como ontogenia. O presente trabalho foi realizado a fim de testar a hipótese de que a dieta e os padrões ecomorfológicos apresentam correlações positivas entre os diferentes estágios de ontogenia de Plagioscion squamosissimus (Heckel, 1840), pois à medida que a espécie passa da fase jovem para adulta altera a sua alimentação e morfologia. A pescada foi escolhida por ser a espécie mais abundante no reservatório da Estação Ecológica de Tapacurá, Estado de Pernambuco. Os padrões ecomorfológicos foram avaliados e relacionados com a alimentação dos indivíduos capturados no reservatório no mês de abril de 2013. As medidas morfométricas foram retiradas de todos os indivíduos capturados e posteriormente recolhidos seus estômagos. Os peixes foram separados de acordo com a fase de desenvolvimento, juvenis e adultos. Também foram tomados seu peso total, comprimento padrão e aferidas onze medidas lineares, utilizadas para obter os índices que representam os atributos ecomorfológicos. Os itens alimentares foram identificados e separados até menor nível taxonômico possível, sendo o mais frequente o camarão Macrobrachium amazonicum (Heller, 1862). Foi possível observar que nos juvenis os itens ingeridos apresentavam maior amplitude no tamanho em comparação com os adultos. Dos dados morfométricos observa-se que os valores de largura da cabeça são maiores nos juvenis e os valores da altura do corpo são mais elevados nos adultos, sendo estes fatores importantes para determinar o tamanho da presa ingerida pelo peixe. Conclui-se que a espécie Plagioscion squamosissimus mostrou relação positiva entre a morfologia e alimentação ao longo do desenvolvimento ontogenético evidenciado pela variação de comprimento dos itens ingeridos entre juvenis e adultos, porém não houve distinção em relação ao alimento ingerido entre eles.

PALAVRAS-CHAVE. Pescada, ecologia trófica, ecomorfologia, Estação Ecológica do Tapacurá.

A ecologia alimentar de uma espécie é consequência de milhares de anos de evolução, refletidos na correlação de suas estruturas morfológicas (Albrecht \& PellegrinICARAMASCHI, 2003), que podem apresentar diferenças em razão das características ambientais e pelo nicho ocupado por cada espécie, com destaque para o nicho alimentar (BAREL, 1983).

A seleção do alimento pelos organismos consiste no tamanho, abundância e estratégias de captura (BROоKS \& Dodson, 1965). Contudo alterações na alimentação podem ser ocasionadas por diversos fatores, destacando-se a ontogenia (GERKING, 1994) e as fases do ciclo reprodutivo (BARBieri et al., 1994; Wootton, 1998). No decorrer do desenvolvimento evolutivo as pressões seletivas, como a competição, estimularam a ampliação de diversas estratégias de forrageamento por parte dos peixes, fazendo com que a segregação trófica se torne muito expressiva (Ross, 1986; Lowe-McconNel, 1999). Essas mudanças de hábito podem ocorrer devido a interações das mudanças nas condições externas, tais como, suprimento alimentar, risco de predação e habitat; e mudanças nas condições internas, como estruturas anatômicas, demandas fisiológicas e comportamentais (LuczKovich et al., 1995).

Estudos que busquem investigar tais relações enquadram-se na área da ecomorfologia, ramo das ciências naturais que estuda a relação entre a morfologia 
e os aspectos ecológicos que permeiam as espécies (Peres-Neto, 1999). As características morfológicas de um determinado grupo de peixes estão diretamente ligadas a sua adaptação e às condições ambientais em que estes se encontram. Desta forma, estas características podem estar ligadas com o tamanho do item alimentar ingerido, agilidade natatória e posição na coluna d'água indicando que a partilha de recursos entre as espécies ocorre por meio da captura de presas de diferentes tamanhos e pela distribuição na coluna d'água (Gomes et al., 2003). Sendo assim, o conhecimento dos aspectos anatomo-funcionais das estruturas morfológicas, permite deduções de padrões ecológicos que são indispensáveis em análises ecomorfológicas de qualquer táxon. Através dos aspectos morfofuncionais pode-se inferir sobre os aspectos ecológicos e ecomorfológicos na biologia das espécies (BREDA et al., 2005).

Plagioscion squamosissimus (Heckel, 1840), conhecida popularmente como "pescada", pertence ao clado Actinopterygii, subclado Perciformes e família Sciaenidae (Fontenele \& Peixoto, 1978; Reis et al., 2003); é originária da Bacia Amazônica e é encontrada em todos os rios e lagos da região nordeste do Brasil (CASATTI, 2005). Foi introduzida em açudes do nordeste no início da década de 1950, pelo DNOCS - Departamento Nacional de Obras Contra a Seca, porém, somente a partir da década de 1970 que $P$. squamosissimus destacou-se na produtividade pesqueira (Fontenele \& Peixoto, 1978; Sato \& Godinho, 1999). É uma espécie neotropical amplamente inserida em águas nacionais, principalmente em ambientes lacustres, como reservatórios (FÉLIX et al., 2009).

Apesar de ser uma espécie introduzida, o que comumente é um problema para a comunidade ictiológica do local, $P$. squamosissimus possui grande valor comercial (Welcomme, 1988; Agostinho et al., 2007); para o estado de Pernambuco ocorre apenas a citação da espécie por Silva Filho et al. (2011) na ictiofauna do reservatório de Duas Unas. Vale destacar que esta é uma das espécies de maior representatividade no Lago Tapacurá (EL-Deir et al., 2012) em São Lourenço da Mata, Pernambuco, localidade de coleta dos exemplares analisados; localmente representa relevância considerável na alimentação das comunidades circunvizinhas.

Estudos relacionados a espécies exploradas são extremamente necessários para o entendimento do seu ciclo de vida, sendo imprescindíveis ao manejo deste recurso pesqueiro (BEGG, 1998), visto que a espécie estudada possui importância econômica gerando renda para as comunidades que necessitam deste recurso para viver.

Somado a isso, é importante avaliar o papel da ecomorfologia como instrumento preditivo de sua ecologia alimentar nas diferentes fases do desenvolvimento e entre espécies de mesmas guildas tróficas. A ecomorfologia pode ajudar a compreender a interação dos peixes com 0 ambiente no qual estão inseridos, sendo utilizada como uma ferramenta eficiente na predição dos hábitos alimentares, partilha de recursos e outros aspectos da ecologia (TEIXEIRA
\& Bennemann, 2007; SAntos et al., 2011).

Diante do exposto, é testado se a dieta e os padrões ecomorfológicos apresentam correlações positivas entre os diferentes estágios de ontogenia, pois à medida que a espécie passa da fase jovem para adulta, altera a sua alimentação e morfologia. Assim, o presente trabalho objetivou definir os padrões ecomorfológicos de juvenis e adultos de Plagioscion squamosissimus, verificando sua relação com o tamanho dos itens alimentares nos diferentes estágios de desenvolvimento.

\section{MATERIAL E MÉTODOS}

A Estação Ecológica do Tapacurá (EET) está situada no município de São Lourenço da Mata, no leste do estado de Pernambuco ( $\left.8^{\circ} 02^{\prime} \mathrm{S} / 35^{\circ} 11^{\prime} \mathrm{W}\right)$ com altitude de 102 $\mathrm{m}$. Apresenta uma área total de aproximadamente 800 ha, dos quais cerca de 400 ha são constituídos por áreas florestadas, circundadas por monocultura de cana-deaçúcar (LyRA-Neves et al., 2007). Atualmente a área da EET é dividida pelas águas do açude de Tapacurá em três remanescentes da Mata Atlântica, denominados de Mata do Toró, Mata do Camocim e Mata da Buchada (LyRA-Neves et al., 2007). Estes fragmentos encontram-se no entorno do Lago Tapacurá, resultante do barramento do rio Tapacurá, construído em 1973 (LyRA-Neves et al., 2007) (Fig. 1). Esse reservatório vem sofrendo intensa ação antrópica, especialmente pelo despejo de poluentes líquidos urbanos, apresentando características eutróficas (CPRH, 2013).

Assim, no Lago Tapacurá foram coletados no mês de abril de 2013 exemplares de P. squamosissimus com o auxílio de uma sequência de redes de espera com malhas de 12, 15, 20, 25, 30, 35, 40, 45, 50 e $60 \mathrm{~mm}$ entrenós adjacentes. Estas redes foram colocadas sempre ao anoitecer e retiradas na manhã seguinte, ficando expostas aproximadamente por 12 horas durante quatro dias, paralelamente próximas à margem em um único ponto. Os espécimes capturados foram colocados em camadas de gelo em escama para anestesiamento e subsequente eutanásia (UFR, 2004). Exemplares testemunhos estão depositados na coleção ictiológica da Universidade Federal Rural de Pernambuco (UFRPE).

Para obtenção dos dados morfométricos, foram aferidas onze medidas lineares, segundo Gatz (1979), WATSON \& BALON (1984) e WinemilLER (1991). Estas foram tomadas do lado esquerdo dos espécimes, com o auxílio de paquímetro digital com 0,01 mm de precisão, sendo elas: comprimento padrão (CP), altura máxima do corpo (AlMCp), largura máxima do corpo (LMCp), altura do pedúnculo caudal (APcd), largura do pedúnculo caudal (LPcd), altura da cabeça (ACb), largura da cabeça (LCb), comprimento da cabeça (CCb), altura do olho (Aio), largura da boca (LB), altura da boca (AB), comprimento do maxilar superior (A) e comprimento do maxilar inferior (B) (Fig. 2). Em seguida foram obtidos 10 índices ecomorfológicos que representam atributos ecomorfológicos designados na Tabela I. Para estimar o tamanho da abertura da boca dos 


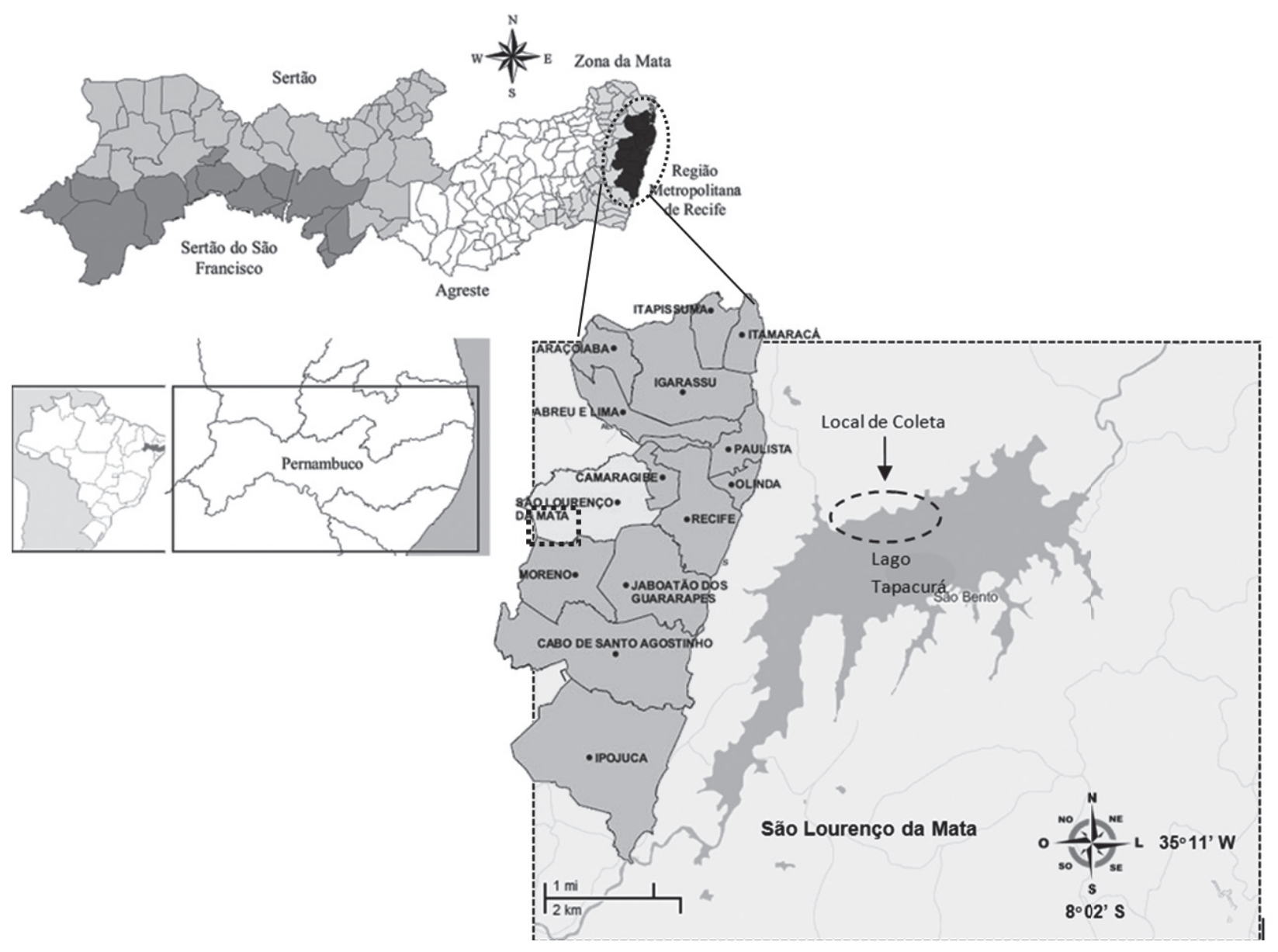

Fig. 1. Mapa da localização do Lago Tapacurá, Estação Ecológica do Tapacurá, Pernambuco, Brasil, com destaque no local de coleta. Fonte: IBGE e Google Earth (adaptado).

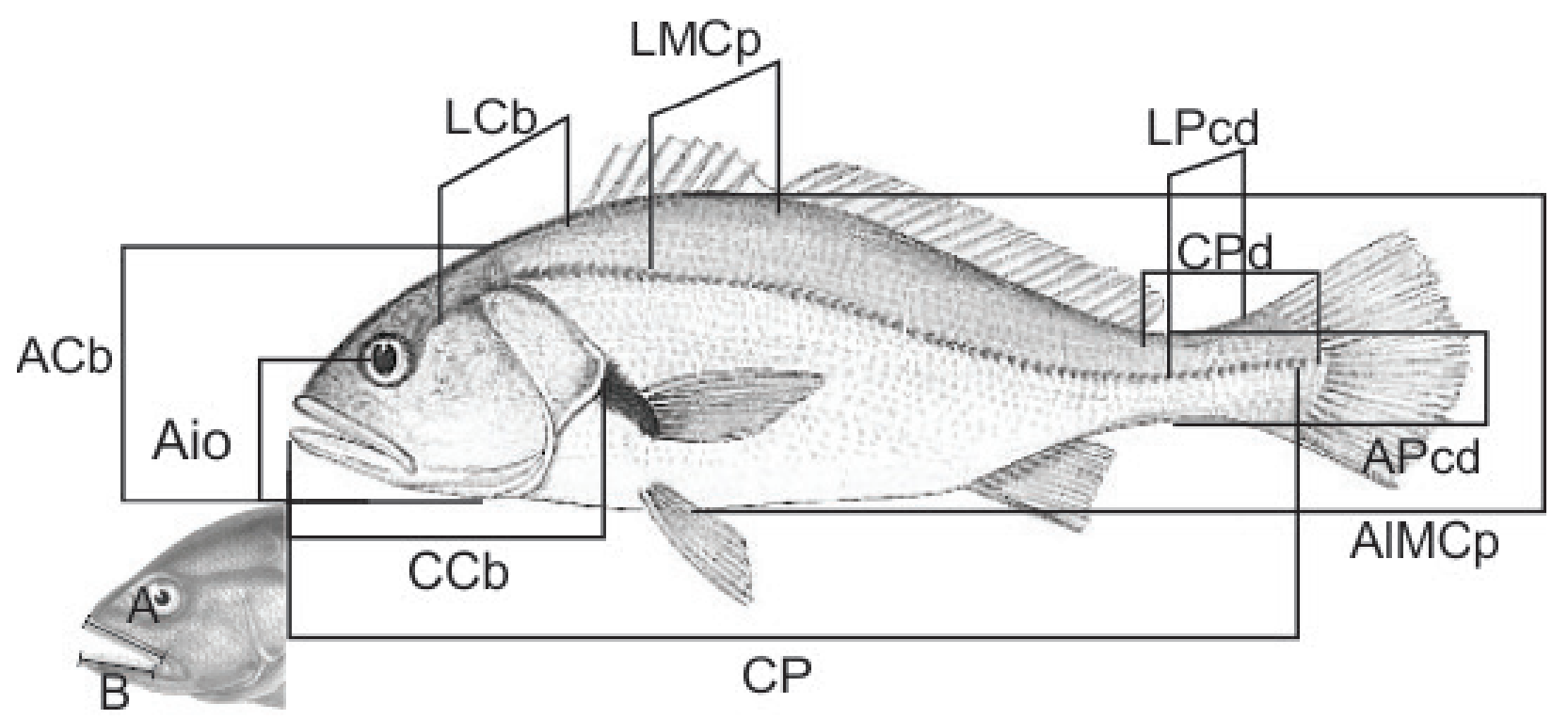

Fig. 2. Medidas morfométricas utilizadas para a pescada Plagioscion squamosissimus Heckel, 1840. 
juvenis, foi utilizada a metodologia proposta por SHIROTA (1970), através da fórmula $D=\sqrt{2} A B$, onde $D=$ tamanho da abertura da boca e $\mathrm{AB}$ é a medida do comprimento da mandíbula superior, baseado nas recomendações constantes em Shirota (1978).

Depois de obtidos dados morfométricos, os peixes foram abertos através de uma incisão ventral longitudinal, a partir do orifício urogenital em direção à cabeça para a retirada das gônadas e estômago. Para análise do conteúdo estomacal foi utilizado o método de frequência de ocorrência dos itens (FO\%) que fornece o espectro alimentar, expresso pela quantidade de estômagos que apresentam um determinado item, em relação ao total de estômagos com conteúdo analisados (ZAVALA-CAMIN, 1996). O conteúdo estomacal foi analisado com auxílio de estereomicroscópio e os itens alimentares identificados até o menor nível taxonômico possível, com base em literatura especializada (Mugnai et al., 2010).

Todas as medidas foram correlacionadas com $\mathrm{CP}$ segundo a relação $\mathrm{Y}=\mathrm{A}+\mathrm{BX}$. A análise foi realizada para duas faixas de comprimento, 24 a $153 \mathrm{~mm}$ e 160 a $400 \mathrm{~mm}$, que corresponde ao tamanho de transição da fase juvenil para a adulta em Plagioscion squamosissimus. Esta correlação visou averiguar diferenças na taxa de variação de tamanho das estruturas corpóreas entre as duas fases de desenvolvimento, mediante a comparação das respectivas equações de regressão linear simples, através do teste t segundo ZAR (1996). Foi analisada a correlação entre $\mathrm{D}$ e o tamanho das presas ao longo das duas fases de desenvolvimento, considerando que o tamanho teórico da maior largura das presas corresponde a $50 \%$ de D (SHIROTA, 1978).

Os atributos ecomorfológicos foram organizados em uma matriz de correlação e submetidos a uma análise

Tab. I. Atributos ecomorfológicos segundo GATz (1979), WATSON \& BALON (1984) e WinemilLER (1991) (LRCb, largura da relativa da cabeça; ARCb, altura relativa da cabeça; $\mathrm{CRCb}$, comprimento relativo da cabeça; LRBo, largura relativa da boca; $\mathrm{ABo}$, altura relativa da boca; LRPd, largura relativa do pedúnculo; LMCp, largura máxima do corpo; CPd, comprimento do pedúnculo caudal; AlPd, altura do pedúnculo caudal; Aio, altura do olho).

\begin{tabular}{|c|c|c|}
\hline & \multicolumn{2}{|c|}{ Índices Ecomorfológicos } \\
\hline \multirow{6}{*}{ 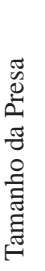 } & Atributos Ecomorfológicos & Fórmulas \\
\hline & Largura Relativa da Cabeça (LRCb) & $\mathrm{LRCb}=\mathrm{LCb} / \mathrm{LMCp}$ \\
\hline & Altura Relativa da Cabeça (ARCb) & $\mathrm{ARCb}=\mathrm{ACb} / \mathrm{AC}$ \\
\hline & Comprimento relativo da cabeça (CRCb) & $\mathrm{CRCb}=\mathrm{CCb} / \mathrm{CP}$ \\
\hline & Largura relativa da boca (LRBo) & $\mathrm{LRBo}=\mathrm{LB} / \mathrm{CP}$ \\
\hline & Altura relativa da boca (ABo) & $\mathrm{ABo}=\mathrm{AB} / \mathrm{CP}$ \\
\hline \multirow{5}{*}{ 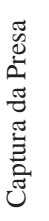 } & Largura relativa do pedúnculo (LRPd) & $\mathrm{LRPd}=\mathrm{LPd} / \mathrm{LMCp}$ \\
\hline & Comprimento relativo do pedúnculo (CRPd) & $\mathrm{CRPd}=\mathrm{CPd} / \mathrm{CP}$ \\
\hline & Altura relativa do pedúnculo (ARPd) & ARPd=AlPd/AlMCp \\
\hline & Índice de compressão do pedúnculo (ICPd) & $\mathrm{ICPd}=\mathrm{AlPd} / \mathrm{LPd}$ \\
\hline & Posição vertical do olho (PVO) & $\mathrm{PVO}=\mathrm{Alo} / \mathrm{AlCb}$ \\
\hline
\end{tabular}

de componentes principais através do Factor Analysis do programa Statistica (7.0) (Tulsa: Statsoft, 2004.) com a finalidade de indicar padrões de semelhança entre os estágios de desenvolvimento.

\section{RESULTADOS}

Foram capturados 43 exemplares de Plagioscion squamosissimus, dos quais 24 foram identificados como juvenis e 19 como adultos. A partir da análise de componentes principais foram selecionados os dois primeiros eixos com autovalores maiores que 0,7 (Tab. II), que concentraram $40,94 \%$ da variabilidade total, sendo PC1 responsável por explicar 22,05\% e PC2 correspondendo a $18,89 \%$. Ao analisar os eixos separadamente, foi possível observar que o atributo mais relevante na PC1 foi a largura relativa da cabeça (LRCb), no qual foi possível visualizar maiores valores para juvenis em relação aos adultos.

As regressões lineares realizadas entre as relações corporais para todos os indivíduos apresentaram correlação positiva, sendo os coeficientes de correlação linear de Pearson ( $\mathrm{r}$ ) todos superiores a $0,7(\mathrm{p}<0,05)$. Através do teste $t$, foi possível observar diferenças significativas entre os estágios de desenvolvimento nas fases juvenil e adulto entre os atributos ecomorfológicos $\mathrm{CCb}, \mathrm{AB}$ e $\mathrm{D}(\mathrm{p}<0,05)$ (Tab. III).

Através desta análise, pode-se observar que as relações de corporais de CCb, AB, e D apresentaram uma tendência de crescimento diferenciada, demonstrando na maioria das proporções corporais maiores nos indivíduos da fase de desenvolvimento juvenil (Tab. IV, Figs 6, 7).

Dentre os estômagos avaliados, $25,58 \%$ encontravam-se sem alimento e $74,42 \%$ continham alimento; após análise foi observada a ocorrência de três itens, divididos em quatro categorias, sendo elas camarão identificado como Macrobrachium amazonicum, partes de camarão (M. amazonicum), Ostracoda e partes de peixe. Através da análise da frequência de ocorrência (FO\%) evidenciou-se a predominância da categoria M. amazonicum com $87,50 \%$, seguido por M. amazonicum (partes) $6,25 \%$, 


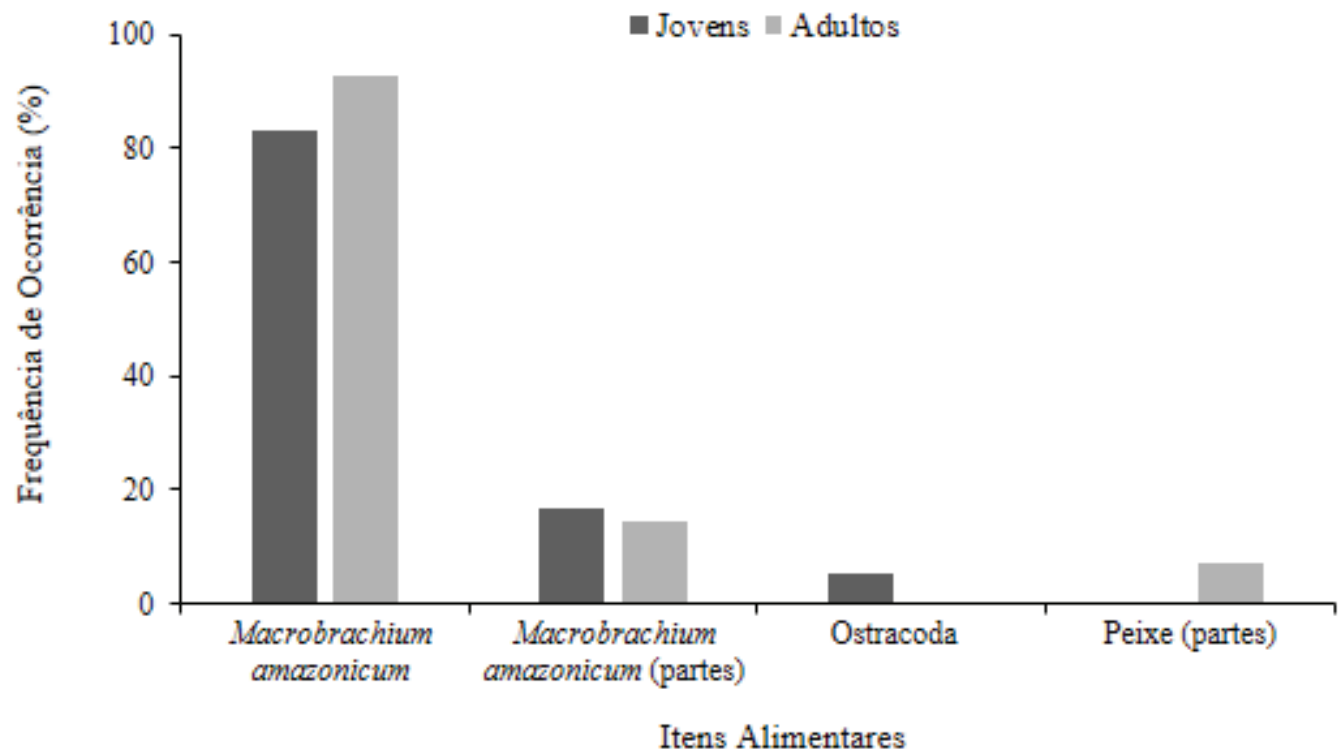

Fig. 3. Frequência de ocorrência (\%) dos itens alimentares consumidos por P. squamosissimus Heckel, 1840 no Lago Tapacurá, Pernambuco, Brasil em abril de 2013.
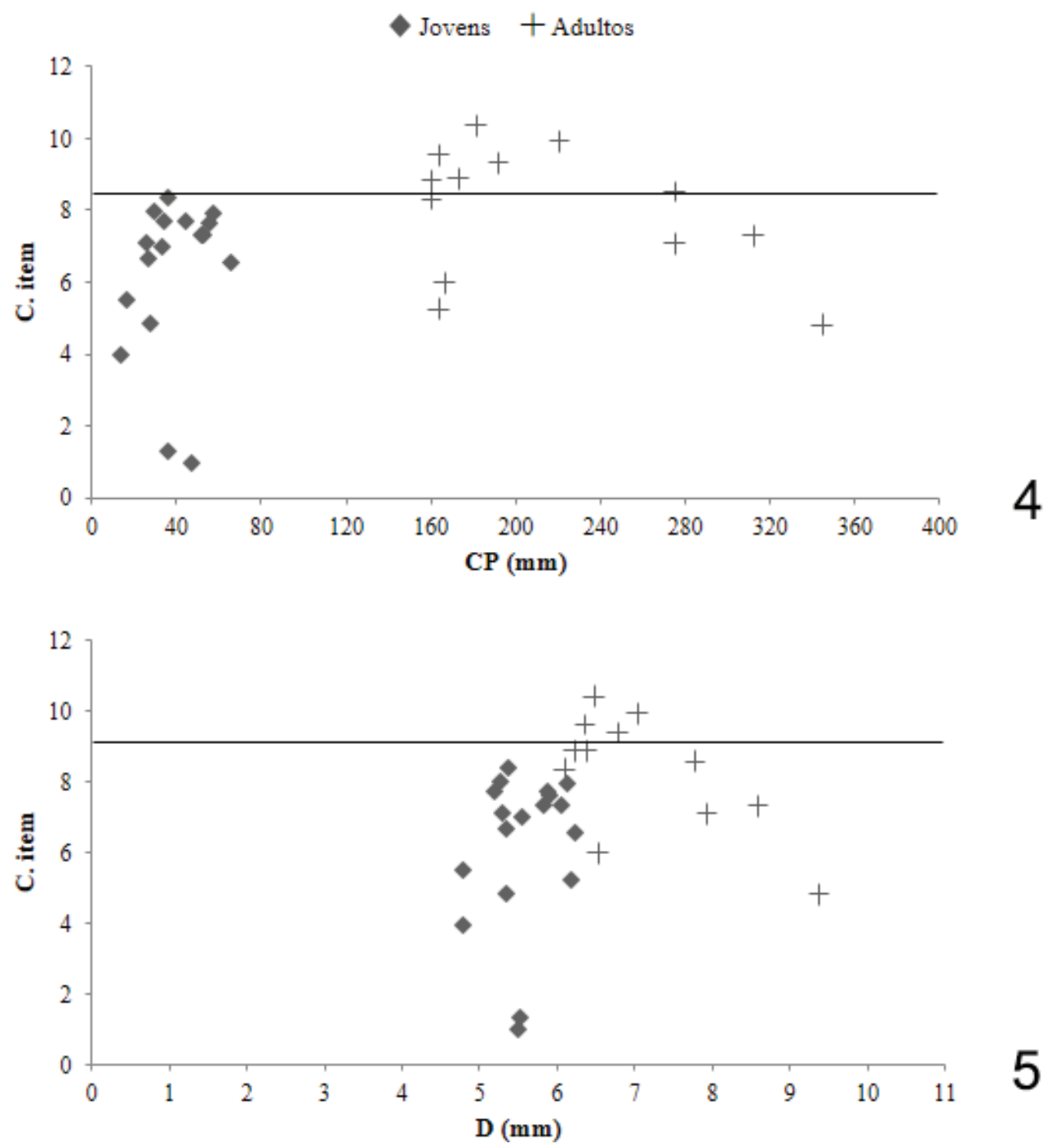

Figs 4, 5. Relações entre o tamanho dos itens alimentares com o comprimento padrão (CP) (4) e o tamanho da abertura de boca (D) (5) de $P$. squamosissimus Heckel, 1840 do lago Tapacurá, Pernambuco, Brasil em abril de 2013. 
Tab. II. Análise dos componentes principais para os dois primeiros eixos gerados, PC1 e PC2, onde os valores em negrito são aqueles que mais contribuíram para a variação.

\begin{tabular}{lcc}
\hline Atributos & PC 1 & PC 2 \\
\hline LRCb & $\mathbf{0 , 7 6 4 1 4 7}$ & $-0,069123$ \\
ARCb & 0,373523 & 0,676995 \\
CRCb & 0,596354 & 0,048511 \\
LRBo & 0,508068 & $-0,299012$ \\
Abo & 0,380711 & 0,522646 \\
LRPd & 0,698339 & 0,077393 \\
CRPd & $-0,192219$ & 0,114277 \\
ARPd & $-0,284322$ & 0,687857 \\
ICPd & $-0,456601$ & $-0,204188$ \\
PVO & $-0,207066$ & $-0,182838$ \\
Variância (\%) & 22,05 & 18,89 \\
Variância Total (\%) & & 40,94 \\
\hline
\end{tabular}

Tab. III. Valores médios das relações corporais de jovens e adultos de Plagioscion squamosissimus Heckel, 1840 (t - teste de Student, * $\mathrm{p}<0,005)$.

\begin{tabular}{lccc}
\hline Medida & Jovem & Adulto & $\mathrm{t}$ \\
\hline CCb & 29,64 & 28,54 & 3,27 \\
ACb & 23,09 & 22,74 & 0,53 \\
LCb & 13,31 & 12,89 & 1,77 \\
A & 12,28 & 11,91 & 1,98 \\
B & 9,16 & 8,79 & 1,07 \\
LB & 13,94 & 14,26 & $-1,2$ \\
AB & 17,24 & 13,37 & 5,95 \\
AlMCp & 28,3 & 28,79 & $-1,1$ \\
LMCp & 12,72 & 12,99 & $-0,85$ \\
LPcd & 4,08 & 4,12 & $-0,33$ \\
APcd & 8,3 & 8,29 & 0 \\
Aio & 11,06 & 10,38 & 1,9 \\
D & 4,55 & 3,28 & 8,72 \\
\hline
\end{tabular}

Tab. IV. Parâmetros da regressão linear entre as relações corporais de Plagioscion squamosissinus Heckel, 1840 para as fases juvenil e adulto (n, número de indivíduos; A, coeficiente linear; B, coeficiente angular; GL, grau de liberdade; r, coeficiente de correlação de Pearson; t, teste de Student, * $\mathrm{p}<0,001)$.

\begin{tabular}{lccccc} 
& \multicolumn{5}{c}{ Fase juvenil } \\
\cline { 2 - 6 } Relações & $\mathrm{n}$ & $\mathrm{A}$ & $\mathrm{B}$ & $\mathrm{r}^{*}$ & $\mathrm{GL}$ \\
\hline CCb/CP & $\mathbf{2 4}$ & $\mathbf{2 , 6 5 7}$ & $\mathbf{0 , 2 9 5}$ & $\mathbf{0 , 9 6 9}$ & $\mathbf{2 2}$ \\
ACb/CP & 24 & 8,313 & 0,226 & 0,822 & 22 \\
LCb/CP & 24 & 2,087 & 0,132 & 0,942 & 22 \\
A/CP & 24 & 0,851 & 0,123 & 0,962 & 22 \\
B/CP & 24 & 0,759 & 0,091 & 0,838 & 22 \\
LB/CC & 24 & 2,225 & 0,138 & 0,948 & 22 \\
AB/CP & $\mathbf{2 4}$ & $\mathbf{5 , 5 4 2}$ & $\mathbf{0 , 1 7}$ & $\mathbf{0 , 8 4}$ & $\mathbf{2 2}$ \\
AlMCb/CP & 24 & 2,133 & 0,282 & 0,953 & 22 \\
LMCp/CP & 24 & 0,378 & 0,127 & 0,907 & 22 \\
LPcd/CP & 24 & 0,04 & 0,119 & 0,907 & 22 \\
APcd/CP & 24 & 0,666 & 0,083 & 0,963 & 22 \\
Aio/CP & 24 & 0,875 & 0,111 & 0,851 & 22 \\
D/CP & $\mathbf{2 3}$ & $\mathbf{2 , 4 7 9}$ & $\mathbf{0 , 0 4 4}$ & $\mathbf{0 , 9 6 6}$ & $\mathbf{2 1}$ \\
\hline & & & & & \\
\hline
\end{tabular}

\begin{tabular}{cccccc}
\multicolumn{5}{c}{} & \multicolumn{3}{c}{ Fase adulto } & \multirow{2}{*}{ t $^{*}$} \\
\cline { 1 - 4 } $\mathrm{n}$ & $\mathrm{A}$ & $\mathrm{B}$ & $\mathrm{r}$ & $\mathrm{GL}$ & \\
\hline $\mathbf{1 9}$ & $\mathbf{0 , 5 8 6}$ & $\mathbf{0 , 2 8 4}$ & $\mathbf{0 , 9 9 5}$ & $\mathbf{1 7}$ & $\mathbf{2 , 9 5 5}$ \\
19 & 1,409 & 0,226 & 0,981 & 17 & 0,067 \\
19 & 1,231 & 0,129 & 0,976 & 17 & 0,817 \\
19 & 0,255 & 0,119 & 0,987 & 17 & 1,755 \\
19 & 0,215 & 0,087 & 0,909 & 17 & 0,961 \\
19 & 1,902 & 0,144 & 0,98 & 17 & 1,334 \\
$\mathbf{1 9}$ & $\mathbf{6 , 7 8 4}$ & $\mathbf{1 , 2 8 7}$ & $\mathbf{0 , 7 9 6}$ & $\mathbf{1 7}$ & $\mathbf{4 , 7 3}$ \\
19 & 4,005 & 0,29 & 0,986 & 17 & 0,091 \\
19 & 2,035 & 0,131 & 0,966 & 17 & 0,94 \\
19 & 0,041 & 0,988 & 0,971 & 17 & 0,681 \\
19 & 0,309 & 0,082 & 0,981 & 17 & 0,234 \\
19 & 0,792 & 0,103 & 0,943 & 17 & 1,891 \\
$\mathbf{1 9}$ & $\mathbf{3 , 7 5 1}$ & $\mathbf{0 , 0 3}$ & $\mathbf{0 , 9 8 9}$ & $\mathbf{1 7}$ & $\mathbf{8 , 7 2 3}$ \\
\hline
\end{tabular}

Ostracoda 3,13\% e Peixe (partes) com 3,13\% (Fig. 3). Entre indivíduos juvenis e adultos não houve diferença quanto ao item alimentar consumido, sendo, portanto Macrobrachium amazonicum o item alimentar mais frequente na dieta em ambas as fases no reservatório Tapacurá (Fig. 3). O tamanho da abertura de boca (D) dos indivíduos de $P$. squamosissimus variou de 4,39 a 6,23 mm nos juvenis e 6,11 a 9,63 mm nos adultos. Em relação ao comprimento dos itens alimentares ingeridos, observou-se maior amplitude na fase de desenvolvimento juvenil $(0,97$ a $8,36 \mathrm{~mm})$, enquanto que os adultos apresentaram uma variação menor $(4,81$ a $10,39 \mathrm{~mm})($ Figs 4, 5). O comprimento do item esteve presente em indivíduos com o tamanho da abertura de boca (D) de 4,39 a 6,23 mm para juvenis e 6,11 a 9,63 mm para adultos. Tais dados correlacionados mostram essa relação (Figs 6, 7).

\section{DISCUSSÃO}

A espécie apresentou características de carnívora nectônica com predominância de camarão em sua dieta, corroborando com Teixeira \& Bennemann (2007), que observaram que valores altos dos atributos relacionados ao tamanho da cabeça, conferem capacidade de capturar itens alimentares relativamente grandes. Embora a captura de presas maiores sejam mais evidentes nos adultos, os quais apresentaram valores de largura relativa da boca menor que os juvenis, os jovens apresentaram maior variação no 


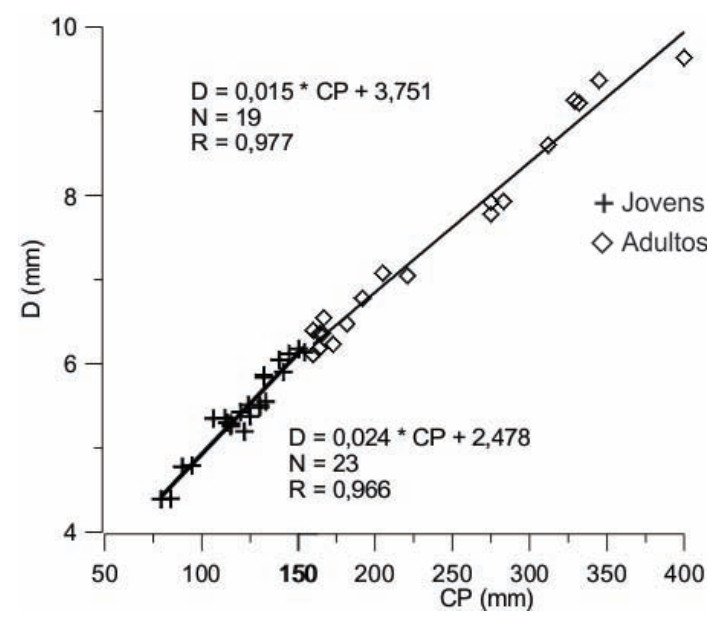

Fig. 6. Relações corporais entre o comprimento padrão (CP) e o tamanho da abertura de boca (D) de P. squamosissimus do lago Tapacurá em abril de 2013.

tamanho dos itens alimentares consumidos pela espécie.

Essa maior amplitude no tamanho dos itens consumidos está relacionada à abertura da boca, medida tal, obtida através do coeficiente " $\mathrm{D}$ " calculado, onde estes foram diferentes significativamente, apresentando maiores valores para os juvenis em relação aos adultos. Segundo Magnhagen \& Heibo (2001), a dimensão da abertura da boca do predador e a altura do corpo da presa, são os fatores fundamentais que determinam se um peixe, com abertura de boca limitada, pode ingerir determinado recurso. De acordo com ZAVALA-CAMIN (1996), o tamanho do item alimentar tem relação direta com o tamanho do predador, visto que a maioria dos peixes ingere sua presa inteira, existindo um tamanho máximo do item em relação ao tamanho da boca do predador.

Apesar de apresentar diferença no tamanho, o item ingerido por ambos estágios de desenvolvimento foi o mesmo - o camarão Macrobrachium amazonicum -, no qual sua captura possivelmente foi facilitada devido à boca da $P$. squamosissimus ser protrátil, o que contribui em investidas em locais de difícil acesso, como algumas macrófitas (BozZA \& HAHN, 2010). Resultado semelhante no hábito alimentar foi observado por CosTA et al. (2009), no qual a pescada também teve como item predominante de sua dieta M. amazonicum. A abundância relatada deste item pode estar diretamente ligada à introdução de $M$. amazonicum nos reservatórios do Nordeste do Brasil, o qual tem sido um dos principais elementos da pesca comercial na região (ODinETZ Collart, 1993). Aliado às características morfológicas citadas acima, enfatizam ainda mais essa flexibilidade característica de $P$. squamosissimus. Entretanto, em outros estudos em diferentes corpos d'água, caracterizam a espécie como piscívora (BenNemAnn et al., 2006; Stefani \& Rocha, 2009; Bozza \& Hahn, 2010), mesmo assim, em todos estes estudos o item camarão foi muito importante na composição da dieta. STEFANI \& Rocha (2009) relataram que a Plagioscion squamosissimus

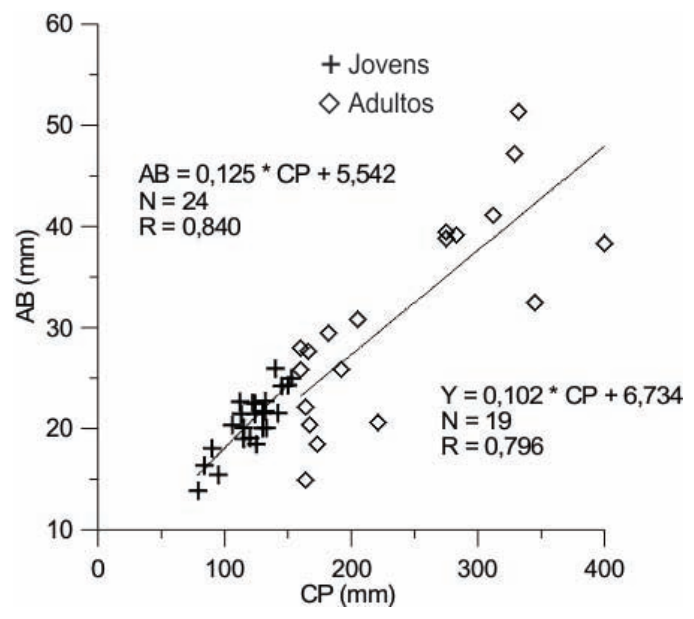

Fig. 7. Relações corporais entre o comprimento padrão (CP) e o comprimento da mandíbula superior (AB) de P. squamosissimus do lago Tapacurá em abril de 2013.

apresenta acentuada plasticidade alimentar, o que garante sua sobrevivência e dominância em muitos reservatórios do Brasil. Essa flexibilidade alimentar evidenciada para espécie, aliado ao oportunismo, são características comuns entre peixes tropicais e subtropicais (ABELHA et al., 2001; Hahn \& Fugi, 2007).

Através da análise de regressão ficou evidenciado que na fase juvenil houve uma maior inclinação $(\mathrm{AB})$ da reta sugerindo que, nesta fase, os indivíduos tendem a apresentar um maior incremento nas estruturas morfológicas analisadas, que nos adultos, tendendo a uma diferença na variação das características morfométricas, de acordo com o estágio de desenvolvimento. Essa análise é reforçada por uma das proposições indicada por KovÁc et al. (1999), que demonstraram que mudanças em caracteres morfométricos podem ser usadas para determinar o tamanho com o qual os peixes passam por transições. Plagioscion squamosissimus apresentou maior proporção do comprimento da maxila superior nos juvenis em detrimento aos adultos, o que reflete diretamente que tamanho de abertura da boca não é um padrão uniforme entre as espécies, conforme demonstrado por MAKRAKIs et al. (2005). Estes autores registraram que as diferenças entre a dimensão da abertura da boca dos indivíduos foi menor em larvas pequenas, aumentando gradativamente junto ao desenvolvimento ontogenético em Plagioscion squamosissimus, Iheringichthys labrosus (Lütken, 1874) e Hypophthalmus edentatus Spix Agassiz, 1829.

Com este estudo foi possível concluir que no local estudado Plagioscion squamosissimus consome predominantemente camarão, independentemente do seu estágio de vida, sendo também encontrados ocasionalmente pedaços de peixes e alguns microcrustáceos. Foi evidenciado que existe uma relação entre morfologia e alimentação, ressaltada através da abertura da boca, onde os juvenis apresentaram maior variação de comprimento dos camarões consumidos quando comparados aos adultos. 
Agradecimentos. Ao Programa de Pós-Graduação em Ecologia - UFRPE pelo apoio e oportunidade da realização da amostragem e Estação Ecológica do Tapacurá representada por Paulo Martins que nos recebeu com muita atenção e carinho.

\section{REFERÊNCIAS BIBLIOGRÁFICAS}

Abelha, M. C. F.; Agostinho, A. A. \& Goulart, E. 2001. Plasticidade trófica em peixes de água doce. Acta Scientiarum 23(2):425-434.

Agostinho, A. A.; Gomes, L. C. \& Pelicice, F. M. 2007. Ecologia e Manejo de Recursos Pesqueiros em Reservatórios do Brasil. Maringá, EdUEM. 501p.

Albrecht, M. P. \& Pellegrini-Caramaschi, E. 2003. Feeding ecology of Leporinus taeniofasciatus (Characiformes: Anostomidae) before and after installation of a hydroeletric plant in the upper Rio Tocantins, Brazil. Neotropical Ichthyology 1(1):53-60.

Barbieri, G.; Peret, A. C. \& Verani, J. R. 1994. Notas sobre alimentação do trato digestivo ao regime alimentar em peixes da região de São Carlos (SP). Quociente Intestinal. Revista Brasileira de Biologia 54(1):63-69.

BAREL, C. D. N. 1983. Towards a constructional morphology of the cichlid fishes (Teleostei, Perciformes). Netherlands Journal Zoology 33:357-424.

BEGG, G. A. 1998. Reproductive biology of school mackerel (Scomberomorus queenslandicus) and spotted mackerel (S. muroi) in Queensland east-coast waters. Marine and Freshwater Research 49:261-270.

Bennemann, S. T.; Capra, L. G.; Galves, W. \& Shibatta, O. A. 2006. Dinâmica trófica de Plagioscion squamosissimus (Perciformes, Sciaenidae) em trechos de influência da represa Capivara (rios Paranapanema e Tibagi). Iheringia, Série Zoologia 96(1):115-119.

Bozza, A. N. \& Hahn, N. S.. 2010. Uso de recursos alimentares por peixes imaturos e adultos de espécies piscívoras em uma planície de inundação neotropical. Biota Neotropica 10:217-226.

BredA, L.; FonTes, E. \& Goulart E. 2005. Ecomorfologia de locomoção de peixes com enfoque para espécies neotropicais. Acta Scientiarum, Biological Sciences 27(4):371-381.

BRooks, J. L. \& Dodson, S. I. 1965. Predation body size and composition of plankton. Science 150:28-65.

CAsatti, L. 2005. Revision of the South American freshwater genus Plagioscion (Teleostei, Perciformes, Sciaenidae). Zootaxa 1080:3964.

Costa, S. A. G. L.; Peretti, D.; Pinto Júnior, J. E. M.; Fernandes, M. A. \& GURGel JúNIOR, A. M. 2009. Espectro alimentar e variação sazonal da dieta de Plagioscion squamosissimus (Heckel, 1840) (Osteichthyes, Sciaenidae) na lagoa do Piató, Assu, Estado do Rio Grande do Norte, Brasil. Acta Scientiarum, Biological Sciences 31(3):285-292.

CPRH. 2013. Relatório de monitoramento da qualidade da água da bacia hidrográfica do Rio Capibaribe Corpo d'água Tapacurá. Disponível em: $<\mathrm{http}: / /$ www.cprh.pe.gov.br/monitoramento/bacias_ hidrograficas/resultados_monitoramento_bacias/bacia_do_rio_capi baribe $/ 39742 \% 3 \mathrm{~B} 37536 \% 3 \mathrm{~B} 17020513 \% 3 \mathrm{~B} 0 \% 3 \mathrm{~B} 0$. asp $>$. A Acessado em: 10.06.2013.

El-Deir, A. C. A.; Silva, K. M. S.; Collier, C. A.; Almeida Neto, M. S., BRUNKEn, H. \& SEVERI, W. 2012. Ictiofauna da Estação Ecológica de Tapacurá. In: Júnior, S. M. A.; Moura, G. J. B. \& El-Deir, A. C. A. eds. Estação Ecológica de Tapacurá: Uma proposta de manejo e conservação. Recife, NUPEEA. p.213-231.

Félix, R. T. S.; Severi, W.; SAntos, A. J. G.; El-Deir, A. C. A.; Soares, M. G. \& Neto, J. E. 2009. Desenvolvimento ovariano de Plagioscion squamosissimus (Heckel, 1840) (Actinopterygii, Perciformes), no reservatório de Pedra, Rio de Contas, Bahia. Biota Neotropica 9(3):131-136.

Fontenele, O. \& Peixoto, J. T. 1978. Análise dos resultados de introdução da pescada do Piauí, Plagioscion squamosissimus (Heckel,1840), nos açudes do nordeste. Boletim Técnico DNOCS 36:85-112.

GATZ JR., A. J. 1979. Ecological morphology of freshwater stream fishes. Talune Studies in Zoology and Botany 21(2):91-124.

Gerking, S. D. 1994. Feeding ecology of fish. San Diego, Academic Press. 416p.
Gomes, L. N.; Pinheiro- Júnior, J. R. \& Piorski, N. M. 2003. Aspectos ecomorfológicos da comunidade de peixes do estuário do rio Anil, Ilha de São Luís - MA. Boletim do Laboratório de Hidrobiologia 16:29-36.

HaHN, N. S. \& Fugi, R. 2007. Alimentação de peixes em reservatórios brasileiros: alterações e consequências nos estágios iniciais do represamento. Oecologia Brasiliensis 11:469-480.

Kovác, V.; Copp, G. H. \& Francis, M. P. 1999. Morphometry of the stone loach, Barbatula barbatula: do mensural characters reflect the species' life history threshold? Environmental Biology of Fishes 56:105-115.

Lowe-McConNell, R. H. 1999. Estudos Ecológicos de Comunidades de Peixes Tropicais. São Paulo, EDUSP. 534p.

LuczKovich, J. J.; Norton, S. F. \& GiLmore, G. 1995. The influence of oral anatomy on prey selection during the ontogeny of two percoid fishes, Lagodon rhomboides and Centropomus undecimalis. Environmental Biology of Fishes 44:79-95.

Lyra-Neves, R. M.; Oliveira, M. A. B.; Telino-Júnior, W. R. \& Santos, E. M. 2007. Comportamentos interespecíficos entre Callithrix jacchus (Linnaeus) (Primates, Callitrichidae) e algumas aves de Mata Atlântica, Pernambuco, Brasil. Revista Brasileira de Zoologia 24:709-716.

Magnhagen, C. \& Heibo, E. 2001. Gape size allometry in pike reflects variation between lakes in prey availability and relative body depth. Functional Ecology 15:754-762.

Makrakis, M. C.; Nakatani, K.; Bialetzki, A.; Sanches, P. V.; BaUmgartner, G. \& Gomes, L. C. 2005. Ontogenetic shifts in digestive tract morphology and diet of fish larvae of the Itaipu Reservoir, Brazil. Environmental Biology of Fishes 72:99-107.

Mugnai, R.; Nessimian, J. L. \& Baptista, D. F. 2010. Manual de identificação de macroinvertebrados aquáticos do estado do Rio de Janeiro. Rio de Janeiro, Technical Books. 174p.

Odinetz Collart, O. 1993. Ecologia e potencial pesqueiro do camarãocanela Macrobrachium amazonicum, na Bacia Amazônica. In: Ferreira, E. J. G.; Santos, G. M.; LeÃo, E. L. M. \& Oliveira, L. A. eds. Bases científicas para estratégias de preservação e desenvolvimento da Amazônia. Manaus, Instituto Nacional de Pesquisas da Amazônia. v.2, p.147-166.

Peres-Neto, P. R. 1999. Alguns métodos e estudos em ecomorfologia de peixes de riachos. Oecologia Brasiliensis 6:209-236.

Reis, R. E.; Kullander, S. O. \& Ferraris Jr., C. J. 2003. Check list of the freshwater fishes of South and Central America. Porto Alegre, EDIPUCRS. 742p.

Ross, S. T. 1986. Resource partitioning in fish assemblages: a review of field studies. Copeia 2:352-388.

Santos, F. L.; Camilo, F. L.; Albieri, R. J. \& Araújo, F. G. 2011. Morphological patterns of five fish species (four characiforms, one perciform) in relation to feeding habits in a tropical reservoir in South-eastern Brazil. Journal of Applied Ichthyology 27:1360-1364.

Sato, Y. \& Godinho, H. P. 1999. Peixes da Bacia do rio São Francisco. In: Lowe-Mcconnell, R.H. eds. Estudos ecológicos de comunidades de peixes tropicais. São Paulo, Edusp. p.401-416.

Shirota, A. 1970. Studies on the gape size of fish larvae. Bulletin of the Japanese Society for the Science of Fish 36:35-368.

1978. Studies on the mouth size of fish larvae. II. Specific characteristics of the upper jaw length. Bulletin of the Japanese Society for the Science of Fish 44:1171-1182.

Silva Filho, E. G.; Silva Santana, F. M. \& Severi, W. 2011. Ictiofauna do reservatório de Duas Unas, bacia do rio Jaboatão, Pernambuco: resultados preliminares da composição e estrutura da assembléia. Revista Brasileira de Ciências Agrárias 6(2):351-361.

Statsoft. Statisca: data analysis software sytem: version 7.0. Tulsa: Statsoft, 2004.

Stefani, P. M. \& Rocha, O. 2009. Diet composition of Plagioscion squamosissimus (Heckel, 1840), a fish introduced into the Tietê River system. Brazilian Journal of Biology 69(3):805-812.

Teixeira, I. \& Bennemann, S. T. 2007. Ecomorfologia refletindo a dieta dos peixes em um reservatório no sul do Brasil. Biota Neotropica 7(2):67-76.

UFR (Use of Fishes in Research). 2004. Committee. Guidelines for the use of fishes in research. American Fisheries Society. Bethesda, Maryland. 53p. 
Watson, D. J. \& Balon, E. K. 1984. Ecomorphological analysis of fish taxocenes in rainforest streams of northern Borneo. Journal of Fish Biology 25:371-384.

Welcomme, R. L. 1988. International introductions of inland aquatic species. Rome, FAO Fisheries Technical Papers. 318p.

WinEMILLER, K. O. 1991. Ecomorphological diversification in lowland freshwater fish assemblages from five biotic regions. Ecological Monographs 61:343-365.
Wootton, R. J. 1998. Ecology of teleost fish. The Netherlands, Kluwer Academic Publishers. 386p.

ZAR, J. H. 1996. Biostatistical analysis. Prentice-Hall, Londres. 718p. ZAVALA-CAMIN, L. A. 1996. Introdução aos estudos sobre alimentação natural em peixes. Maringá, Nupelia EDUEM. 129p. 